\title{
Enhancing the EAST-ADL Error Model with HiP-HOPS Semantics
}

\author{
By Septavera Sharvia* \\ Yiannis Papadopoulos ${ }^{\dagger}$ \\ DeJiu Chen ${ }^{*}$ \\ Martin Walker ${ }^{+}$ \\ Wenjing Yuan \\ Henrik Lonn ${ }^{\circ}$
}

\begin{abstract}
EAST-ADL is a domain-specific modelling language for the engineering of automotive embedded systems. The language has abstractions that enable engineers to capture a variety of information about design in the course of the lifecycle - from requirements to detailed design of hardware and software architectures. The specification of the EAST-ADL language includes an error model extension which documents language structures that allow potential failures of design elements to be specified locally. The effects of these failures are then later assessed in the context of the architecture design. To provide this type of useful assessment, a language and a specification are not enough; a compiler-like tool that can read and operate on a system specification together with its error model is needed. In this paper we integrate the error model of EAST-ADL with the precise semantics of HiP-HOPS - a state-ofthe-art tool that enables dependability analysis and optimization of design models. We present the integration concept between EASTADL structure and HiP-HOPS error propagation logic and its transformation into the HiP-HOPS model. Source and destination models are represented using the corresponding XML formats. The connection of these two models at tool level enables practical EAST$A D L$ designs of embedded automotive systems to be analysed in terms of dependability, i.e. safety, reliability and availability. In addition, the information encoded in the error model can be re-used across different contexts of application with the associated benefits
\end{abstract}

\footnotetext{
${ }^{*}$ Research Assistant, University of Hull, UK.

†Professor, University of Hull, UK.

${ }^{*}$ Associate Professor, KTH Royal Institute of Technology, Sweden.

${ }^{+}$Lecturer, University of Hull, UK.

'Chalmers University of Technology,Sweden.

SE, Volvo Technology, Sweden.
} 
for cost reduction, simplification, and rationalisation of dependability assessments in complex engineering designs.

\section{Introduction}

Model-based engineering has become increasingly important in managing the ongoing advances in functionality and complexity of modern safety-critical embedded systems. There is a growing development in domain-specific modelling languages to support the design lifecycle from the early requirements stage up to the detailed hardware and software designs. Such languages aim to address the challenges arising from the management of nominal system design, design refinement and evolution, as well as the safety requirements and error behaviours.

EAST-ADL (EAST-ADL, 2014) is an Architecture Description Language (ADL) which provides an integrated and systematic support for modelling of automotive systems. EAST-ADL facilitates multi-level abstractions which allow design and engineering concerns to be better managed. The specification of the EAST-ADL includes an error model which describes potential failures of the design elements. Specifications using the error model can be used to assess of the effects and propagations of these failures through the system. To perform this assessment, however, a connection between the modelling language and a model-based safety analysis tool needs to be established.

Consideration for safety and reliability has been crucial throughout the development of safety critical systems. Various model-based safety analysis techniques (Joshi et al., 2006; Sharvia \& Papadopoulos, 2011) have been proposed and developed throughout recent years. These techniques can be based on architectural decomposition of the system, e.g. the HiP-HOPS technique (Papadopoulos, et al., 2001), or they may be based on formal verification. such as the FSAP-NuSMV model checking approach (Bozzano \& Villafiorita, 2006). Both categories of technique aim to identify the causes of failures, derive and refine safety requirements, and assist in the development of safety solutions. Some techniques have been extended with additional capabilities, e.g. HiP-HOPS is also capable of architectural optimisation and automatic allocation of safety requirements.

Integration between emerging domain-specific languages and a mature model-based safety analysis technique will be greatly beneficial in enabling robust and consistent automotive system development and assessment. In this paper, we extend the error model of EAST-ADL with the semantics of HiPHOPS to enable safety analysis to be performed on EAST-ADL models. This extension will pave the road for exploiting many of the capabilities provided by HiP-HOPS, including reliability analysis, availability analysis, safety analysis through Fault Tree Analysis (FTA) and Failure Modes and Effects Analysis (FMEA), multi-objective optimization, and safety requirement allocation, all using data gathered from the EAST-ADL model. The information contained in the EAST-ADL error model can be re-used across different contexts of 
application with the associated benefits of cost reduction, simplification and rationalisation of dependability assessments in complex engineering designs.

The integration requires translation of the models in the automotive domain to models in the safety analysis domain, i.e., a transformation of the EAST-ADL error model to a corresponding HiP-HOPS model. The concrete source and destination models are both represented in XML-based formats, which are EAXML and HiP-HOPS XML respectively. A translator tool has been developed to perform the transformation between these models. This involves a conceptual semantic mapping between the domains as well as representation of the output in the target concrete syntax (i.e. HiP-HOPS). The connection of these two models at tool level enables various dependability analyses to be performed on practical EAST-ADL designs in an incremental and iterative manner.

The remainder of this paper is structured as follows: Section 2 discusses the different levels of abstraction in EAST-ADL, and their contribution to the design lifecycle. The Error Model is introduced and the main elements of the Error Model are briefly discussed. Section 3 discusses the main processes in HiP-HOPS, as well as its contribution in providing automated safety and reliability analysis. The main elements of the HiP-HOPS XML interface are also presented. Section 4 presents the semantic mapping between EAST-ADL and HiP-HOPS models. Then, the translation process and algorithm are explained. Section 5 of this paper presents a small case study to illustrate the transformation. Section 6 presents our conclusions and outlines future works.

\section{EAST-ADL}

EAST-ADL provides a comprehensive approach for the modelling of automotive embedded systems. It captures requirements in a standardized form and covers design aspects such as vehicle features, analysis functions, communications, and software and hardware components. EAST-ADL contains multiple layers of abstractions which allow the system to be modelled from different viewpoints. The layers of abstractions are illustrated in the figure below. This provides separation-of-concerns and enables effective quality management. It also provides traceability relations which allow an element to be traced from feature to components in hardware and software. The four principle abstraction layers are the Vehicle level, Analysis level, Design level and Implementation level (Blom, et al., 2012). 
Figure 1. EAST-ADL Abstraction Level

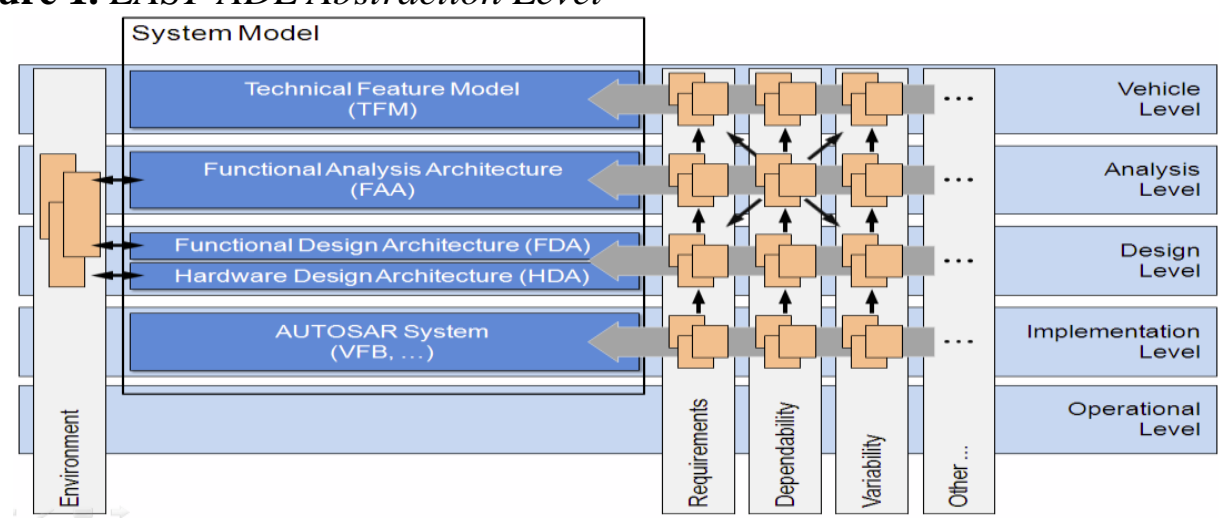

The Vehicle level model provides the top-level definition of an embedded system by capturing its externally visible functionality. The Technical Feature Model at this level represents the content and property of the vehicle, without going into its realization. For dependability engineering, this top-level system description becomes the basis of the analysis of the safety objectives, for example via Preliminary Hazard Analysis (PHA).

The Vehicle level is later refined at the Analysis level. The Functional Analysis Architecture (FAA) at this level captures the underlying system input and output functions as well as the control functions. It does not consider any implementation details. For dependability engineering, the Functional Analysis Architecture becomes the basis for the assessment of functional anomalies. System safety objectives are mapped into more detailed functional safety requirements.

The Analysis level is then refined for system realization, and the choices of technologies for computation and communication are considered. This implementation-oriented aspect is covered at the Design level. System models at this level typically consist of a Functional Design Architecture (HDA) and Hardware Design Architecture (HDA). The Functional Design Architecture defines the grouping and partitioning of functions, and takes into account aspects such as efficiency, legacy of usage, reusability, and hardware allocations. It also specifies the structure of the system function to be implemented. For dependability engineering, it becomes the basis for the analysis of error behaviours of software and hardware components. This leads to the refinement of functional safety requirements to more detailed technical safety requirements. The Hardware Design Architecture describes the target hardware platform and captures the constraints of the abstract functions to the platform.

The Implementation level provides the specification of software components based on AUTOSAR (AUTOSAR, 2013). Traceability is supported at all levels from Implementation level to Vehicle level. For dependability engineering, the technical safety requirements are further refined into software and hardware requirements. EAST-ADL allows requirements to be traced to the related design solutions, verification and validation cases, and other interconnected requirements. 
EAST-ADL has also been recently extended with a Behavioural Description Annex (Chen, et al., 2013) which captures various behavioural concerns. This includes behavioural modelling and analysis, which are important in requirement engineering, architectural design, and design verification and validation.

\section{Error Model}

EAST-ADL allows safety requirements and concerns (such as hazards, faults, and failures etc) to be modelled and analysed in parallel to the development of the nominal system model. For this, an Error Model Type is introduced to support the annotation and management of error behaviours, and thus allow the traceability from error behaviours to system functions or components.

The composition of the Error Model Type is achieved through the Error Model Prototype. The Error Model Prototype represents the instantiation of an Error Model in a specific context and reflects the composition of the nominal system design. Each Error Model allows description of anomalies in term of estimated faults and failures. A Fault Failure entity is used to declare the actual value of an anomaly, usually with a certain enumeration type, for example \{omission, commission\}. The modelling of failure propagation is facilitated through ports. A Fault InPort describes the faults a target can receive from its environment or other Error Models. a Failure OutPort describes the failures which may occur and propagate to the environment or other Error Models. These ports can be traced to the corresponding communication ports of functions or components in the nominal system architecture.

An Error Model Type may have both Internal Faults and Process Faults. An Internal Fault represents an internal malfunction which causes errors when activated, whereas a Process Fault represents a permanent systematic fault. The Error Model Type may also contain Failure Logic, which is used to describe how output failures of a system function or component occur in relation to its internal faults or faults propagated from its inputs. The formalism used to express the Failure Logic depends on the analysis techniques being applied.

Fault Failure Propagation Links are used to describe error propagations across Error Models. These propagation links connect an output failure port (i.e., Failure OutPort) with an input fault port (i.e., Fault InPort) with shared variable semantics.

Figure 2 illustrates the domain model for the Error Model: 
Figure 2. Domain Model for the EAST-ADL Error Model Type (EAST-ADL, 2014)

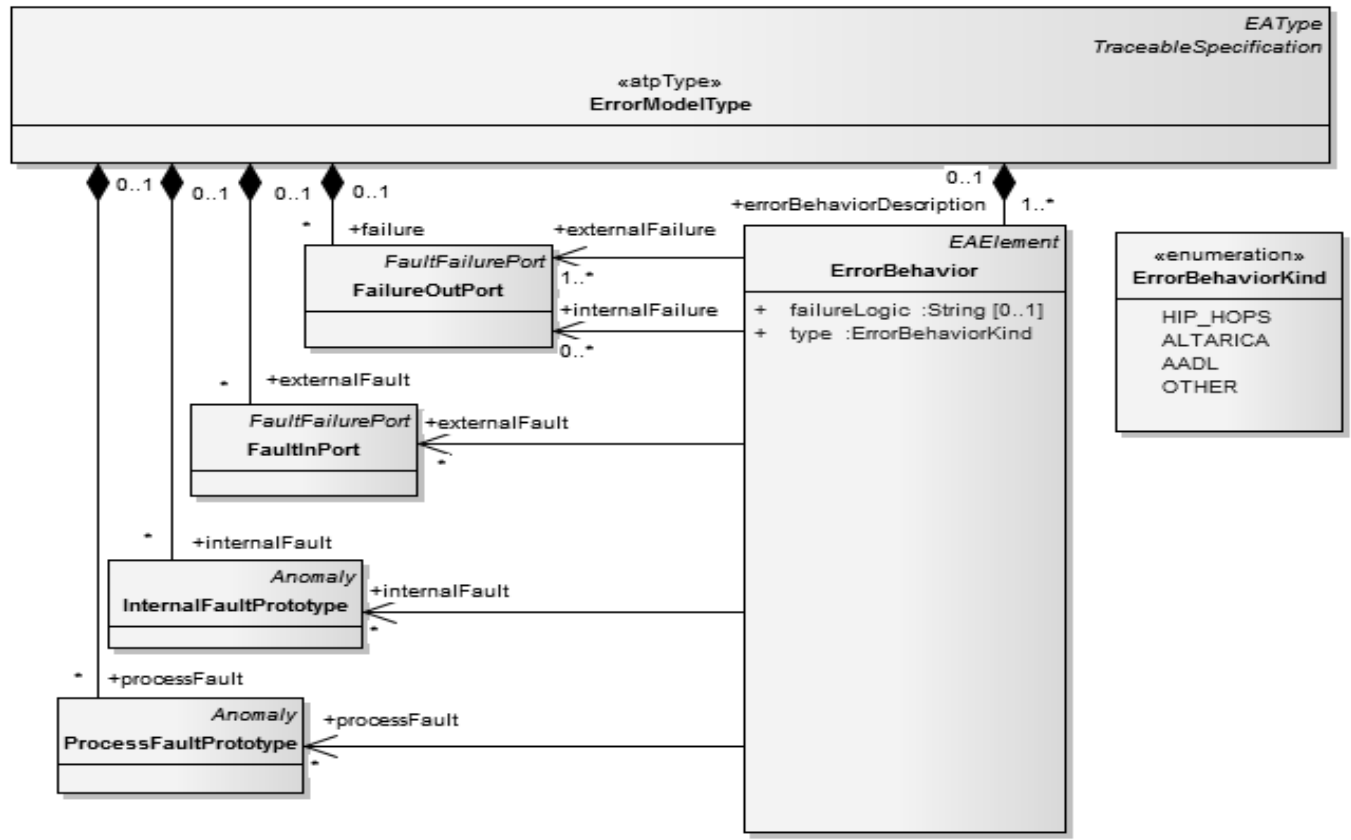

\section{HiP-HOPS}

Hierarchically Performed Hazard Origin and Propagation Studies (HiPHOPS) (Papadopoulos, et al., 2001)is a safety analysis technique which automatically performs Fault Tree Analysis (FTA) and Failure Mode and Effect Analysis (FMEA) on the basis of a system model. HiP-HOPS models the propagation of failures by synthesizing hierarchical component failure logic into a network of fault trees. There are three main phases in HiP-HOPS:

- a model annotation phase, during which the analyst annotates the system architecture with component-level descriptions of failure behaviour;

- a fault tree synthesis phase, during which HiP-HOPS automatically synthesizes system-level fault trees to model the propagation of failures through the system;

- and the analysis phase, during which HiP-HOPS performs FTA and FMEA analyses on the basis of the failure propagation model it has generated.

The model annotation phase provides information on how each individual component can fail. This local failure information takes the form of a set of logical expressions which are manually added to each component. These local failure expressions describe how failures of the component's outputs can be caused by a combination of failures received at the component's inputs and/or by failure modes (internal malfunctions) of the component itself. Common 
cause failures are also supported, as are failures propagated via other means, e.g. from allocated components. In this way it is possible to model more sophisticated scenarios - for instance, the effects on a software function when the hardware processor executing that function fails.

The synthesis phase produces an interconnected network of fault trees which link system-level failures (i.e., failures of the system's output functions) to component-level internal failures by using the model topology and component failure information. These fault trees show how the component failures propagate from one component to another and how ultimately they may affect the wider system, whether individually or in combination with other component failures.

In the analysis phase, the synthesized fault trees are analysed and an FMEA is generated. Both qualitative and quantitative analysis can be performed depending on the amount of information provided. Qualitative analysis is performed to obtain a list of minimal cut sets, which represent the necessary and sufficient combinations of failure modes required to cause every system failure in the model. A multiple failure mode FMEA is also produced, which shows both direct effects of failure modes on the system as well as the further effects of the failure modes (i.e., the effects a failure mode can have on the system when it occurs in conjunction with other failure modes). Results are available in a variety of formats, including spreadsheets and HTML files that can be conveniently displayed through a web browser.

In a design development lifecycle, the automated nature of HiP-HOPS supports an iterative and efficient safety analysis approach. FTA and FMEA are both traditionally performed as a manual process, and in the context of large complex systems, they may become laborious and error-prone. The automatic synthesis of FTA and FMEA information by HiP-HOPS alleviates the pressure for safety analysts and helps ensure that safety analysis results are synchronized with each new iteration of the system design model. The results from these analyses help identify the weak points in the system designs and allow problems to be addressed earlier in the design lifecycle. This contributes to a more robust, consistent, and effective process.

HiP-HOPS can also assist in the making of design decisions by providing capability for multi-objective optimization to achieve more efficient architectural configurations. Design decisions often need to address conflicting requirements, e.g. the requirement to maximize dependability and minimize cost. When multiple possible architectures involving different subsystem configurations and component alternatives can fulfil these various requirements, a large number of different design options become available. Identification of the most dependable design with the lowest cost will therefore involve a large search space, often one too large to be explored manually. Furthermore, there is often no single 'optimal' design solution, especially when requirements conflict (as dependability and cost often do). In this case the designer must find a suitable solution which achieves a satisfactory trade-off between dependability and cost. Multi-objective optimization is valuable in such situations as it rapidly identifies balanced trade-off solutions. HiP-HOPS 
automates this optimization process and therefore helps with the identification of a suitable or optimal architecture design. More discussion on multi-objective optimization can be found in Adachi et al. (2011) and Mian et al. (2014).

\section{HiP-HOPS Error Model}

The HiP-HOPS error model follows the semantics defined in (Walker, et al., 2013). The top most element of the HiP-HOPS error model structure is the Model, which represents all views of the system under analysis and thus encapsulates all other data. Within the Model is a hierarchical structure of subsystems and components, represented in figure 3 below:

Figure 3. Model Representation in HiP-HOPS (Walker, et al., 2013)

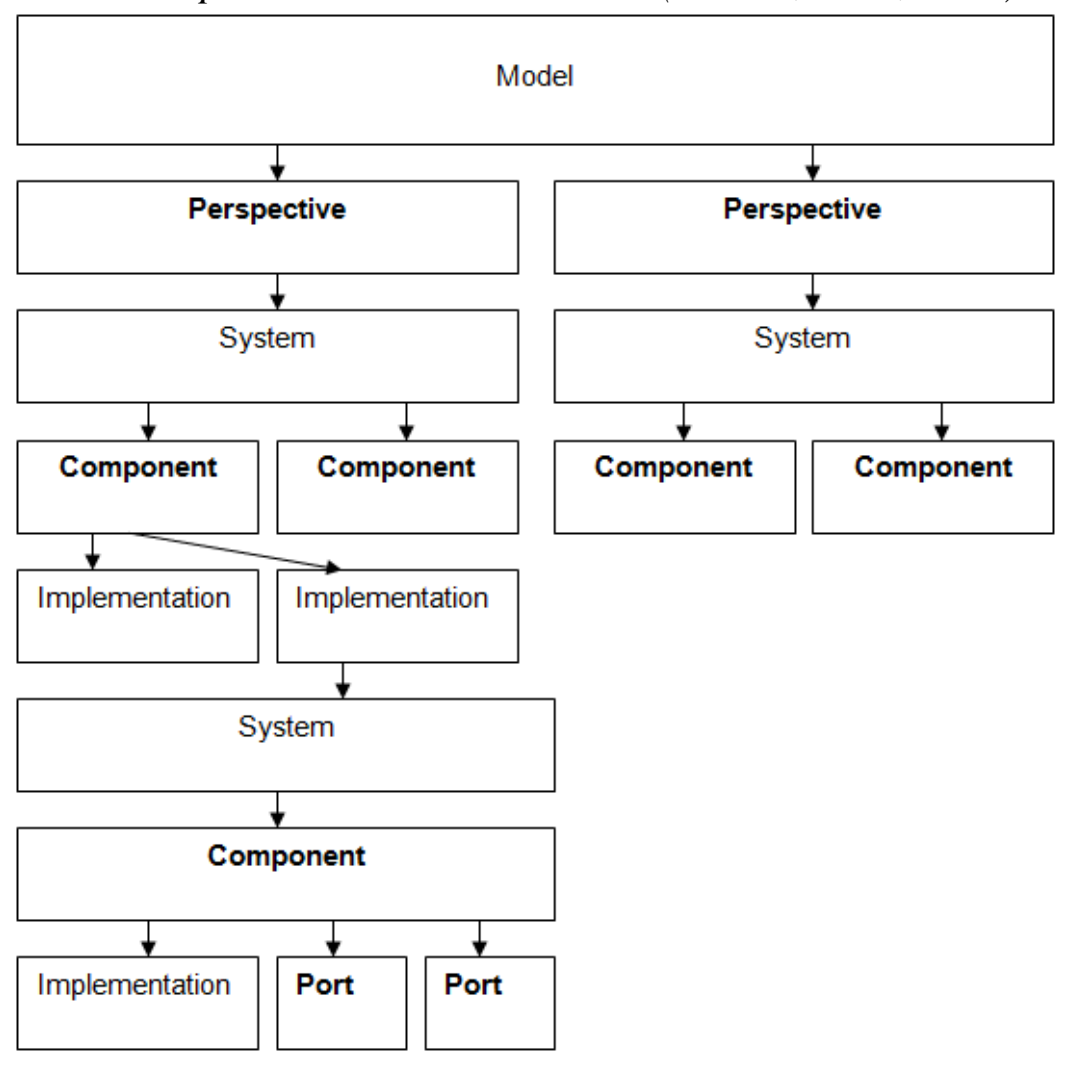

Each Model can contain one or more Perspectives of a system. A Perspective is a particular view of a system. This allows separation of concerns between software and hardware perspectives in the same way that EAST-ADL has a separate FDA and HDA models in its Design level. It is also possible to create a HiP-HOPS model with a single, default perspective containing both hardware and software elements if preferred.

Within each Perspective is a top-level System element, representing the system being studied. The System contains a set of Components and a set of Lines connecting these components together. Components may contain their own System elements (via Implementations), which in turn contain subcomponents, and thus subsystem hierarchies can be represented. 
Components are the main elements of the system hierarchy and describe concrete components or abstract functions in the system.

Connections between Components are modelled by means of Ports and Lines. Each Component has a set of input and output Ports which define the interface for that Component. These Ports can then be connected to Ports of other Components by one or more Lines. Generally speaking, Ports are defined as either inputs or outputs, although HiP-HOPS also supports bi-directional ports (i.e., serving as both inputs and outputs).

A Component must also define one or more Implementations. These represent different concrete implementations or versions of a given component or function, i.e., different ways of fulfilling the functionality defined by the Component's interface. For example, components from different manufacturers could be represented by different Implementations in HiP-HOPS. Different Implementations may have different child Systems, allowing complex alternative hierarchies with different structural or behavioural characteristics to be modelled.

The Implementation is the model element that contains the failure data (and thus different Implementations of a Component may have different failure behaviour as long as it still meets the interface for that Component). In general, failure data is represented as a set of Basic Events and a set of Output Deviations.

Basic Events are the basic failures of a system and typically represent individual component failure modes. They are defined internal to a component and may contain optional quantitative failure information (e.g. failure rates and repair rates).

An Output Deviation represents an error or fault propagated from an output of a component. They are defined by a failure class and a specific output Port; failure classes represent different types of failure possible at a given output. For example, one may have omissions and commissions of a given output, or value failures such as "too high", "too low", "too early", "too late" etc, all associated with each output port.

The other aspect of an Output Deviation is its cause, which is represented by a Boolean expression that links Input Deviations (i.e., failures propagated to the inputs of the Component) and internal failure modes of the component. For example, an Output Deviation "omission of signal" from a given component may be caused by internal failure of that component or a corresponding omission of input to that component: thus "Omission-Signal $=$ InternalFailure OR Omission-Input".

If an Output Deviation is flagged as a system output, then it serves as an Output Deviation for the system as a whole and will act as the starting point of an analysis by becoming the top event of a fault tree. 


\section{Transformation of EAST-ADL Models to HiP-HOPS Models}

\section{Semantic Mapping between EAST-ADL and HiP-HOPS}

The first step in the model transformation process is to establish the semantic mapping between the two models. This enables the entities in the EAST-ADL model to be translated to corresponding elements in the resultant HiP-HOPS model.

The semantic mapping between EAST-ADL and HiP-HOPS is summarized in the following table 1:

Table 1. Semantic Mapping between EAXML and HiP-HOPS XML

\begin{tabular}{|c|c|}
\hline EAST-ADL & HiP-HOPS \\
\hline Error Model Type & System \\
\hline Error Model Type.Parts.Error Model Prototype & System or System.Component \\
\hline Error Model Type. ExternalFault.Fault In Port & System.Component.Ports \\
\hline Error Model Type.Failures. Failure Out Port & System.Component.Ports \\
\hline Error Model Type. & System.Lines \\
\hline FaultFailureConnectors. Propagation Link & $\begin{array}{c}\text { System.Component. } \\
\text { Implementation.FailureData. } \\
\text { Brror Model Type. Internal Fault }\end{array}$ \\
\hline Error Model Type. \\
ErrorBehaviour.ErrorBehaviourDescription.Failure \\
Logic Expression & $\begin{array}{c}\text { System.Component. } \\
\text { Implementation.FailureData. } \\
\text { OutputDeviation }\end{array}$ \\
\hline
\end{tabular}

The transformation concerns a number of different aspects: the structural transformation, transformation of component specific behaviour, and the intercomponent failure propagation. EAST-ADL models and HiP-HOPS models are structurally different. A successful transformation must preserve the semantics of the original model but change the structure of the model to match the target model format. (Biehl, et al., 2010) highlights the fact that EAST-ADL follows the concept of initially declaring types and referencing to the declaration from each point of use; HiP-HOPS however, couples declaration of a type and its usage. In HiP-HOPS, the types are declared at the same point as they are used, and therefore during transformation, the declarations have to be included at every point of usage. This is particularly demonstrated in the transformation between EAST-ADL Error Model Prototype (EMP) and HiP-HOPS Component, in which the Error Model Type (EMT) defines the ports, internal connection, and error behaviour, while the EMP obtains this information from the EMT it was instantiated from. In HiP-HOPS, these are declared for each Component.

\section{Transformation Process}

The transformation process starts by iterating through the list of Error Model Types (EMT) in the EAST-ADL model. One of the user input parameters identifies the top-level EMT which will be used as the basis of the 
analysis. Information on other EMTs are parsed and stored in the model as they will be referenced by an Error Model Prototype (EMP).

Although EMT maps to a HiP-HOPS System in general, the top-level EMT is processed slightly differently. This is because a HiP-HOPS System contains only list of components and the lines defining the connection between these components. The top-level EMT, however, would also contain Fault InPorts and Failure OutPorts. To model this, a top-level Component is created to represent the top-level EMT.

This top-level EMT becomes the basis for the construction of HiP-HOPS System. The EMPs contained within the EMT represent the system decomposition. The referenced EMT for each of these EMP is subsequently checked and if it further contains decomposition, it becomes a subsystem and is processed recursively. If it does not have further decomposition, a HiP-HOPS Component is created. The construction of HiP-HOPS Components requires information on the ports and failure data.

Input and output ports of the Component can be obtained from Fault InPort and Failure OutPort of the referenced EMT. Failure Data in HiP-HOPS is contained as part of the implementation. Implementation contains information about the failure data of the Component. These are represented as HiP-HOPS Basic Events and Output Deviations. The Basic Event is obtained from the EMT Internal Fault, and the Output Deviation is obtained from the EMT Failure Logic. Multiple Failure Logics are parsed into different Output Deviation accordingly.

While this shows the mapping of EMT to HiP-HOPS Component, information on the name of the Component and its connectivity inside the System is obtained from the EMP. This is because unlike the internal structure (ports and internal connectors) and failure information of the referenced EMT, which can be reused, the name and connection of the Component depend on its usage context.

The connectivity of Components inside a HiP-HOPS System is decided by the EMP connections. HiP-HOPS Lines connect Components in the same subsystem together. Each Line element contains a list of Connections which define the connection logic for each port connected to the line. Each Connection has a port name and port Expression. Port Expression is a logical expression containing names of other ports on the line, Boolean expression or specific values. A HiP-HOPS Line and Connection are obtained from the Fault Failure Propagation Link of an EMT. The Fault Failure Propagation Link connects Failure OutPort with Fault InPort. During the transformation, Failure OutPort (source of failure) is mapped to the port expression, and the Fault InPort (destination) is mapped to port.

The transformation algorithm can be briefly summarized as follows:

Create top-level component for top-level Error Model

Create HiP-HOPS System for the EMT 
For every EMP contained in this EMT

Check if the referenced EMT further contains EMP, If yes:

Create HiP-HOPS System for the referenced EMT (recursive). This is a subsystem.

Create HiP-HOPS Component for the EMP, adding the subsystem to the Implementation of Component.

Add the HiP-HOPS Component to HiP-HOPS System.

If not

Create HiP-HOPS Component for the EMP.

Add the HiP-HOPS Component to HiP-HOPS System.

Create HiP-HOPS Lines elements based on Fault Propagation Link

Add Components and Lines to System

Create HiP-HOPS Component

Create HiP-HOPS Port

Create HiP-HOPS Implementation

Add Port and Implementation to Component

Create HiP-HOPS Port

Create HiP-HOPS Implementation

Create HiP-HOPS Basic Event

Create HiP-HOPS Output Deviation

Add Basic Event and Output Deviation to Implementation

Concrete Representations of the Models: EAXML and HIPX

The transformation from the EAST-ADL model to a HiP-HOPS model is performed through the translation between their corresponding concrete representations. EAXML (Blom, 2013) is an XML-based exchange format for EAST-ADL models. The EAXML schema is auto-generated from the EASTADL meta-model, and it contains the serialized form of the EAST-ADL metamodel instances. HIPX is an XML-based exchange format used as input to HiP-HOPS and directly represents the HiP-HOPS model structure described earlier. A successful transformation of EAST-ADL to HiP-HOPS therefore requires transforming an EAXML file into a HIPX file.

A translator tool called EAXML2HIPXML has been developed to perform this transformation. The translator tool was written in Java and constructs internal representations of both models. It performs four main tasks: reading of the EAXML file, constructing an internal representation of the EAST-ADL model, transforming the EAST-ADL model to HiP-HOPS model, and writing the output HiP-HOPS XML file.

Reading the EAXML File

The Java DOM (Document Object Model) API is used for the parsing of the XML documents. It is a hierarchy-based parser that loads the XML content 
into a tree structure and creates an object model of the XML document. The translator tool then creates an internal representation of the EAXML model. It starts by reading through the list of Error Model nodes contained in the model, and for each of these Error Model nodes, information on its Fault InPort, Failure OutPort, Internal Fault, Failure Logic, Error Model Prototype, and Fault Failure Propagation Link is obtained.

Fault InPort, Failure OutPort, and Internal Fault are referenced by unique names. Multiple failure logics are separated by the semicolon ";" delimiter. The Error Model Prototype represents the hierarchical decomposition for the Error Model, and contains a reference to the Error Model it instantiates from. In the EAXML model, this reference contains the full path of the EAST-ADL dependability package. This has been trimmed, and the instantiated Error Model is referenced by Error Model name. The Fault Failure Propagation Link connects Failure OutPorts with Fault InPorts. Both Failure OutPort reference <FROM-PORT-IREF> and Fault InPort reference <To-PORT-IREF> references contain information on the port reference and the Error Model Prototype reference. These are transformed and stored into a simplified form of "Component.Port" which will be easily fitted and recognized in HiP-HOPS model.

Writing the HIPX File

The HiP-HOPS XML Document is produced based on the schema defined in (Walker, et al., 2013). The HiP-HOPS XML file supports all the features and capabilities of HiP-HOPS. The HiP-HOPS XML file can also be zipped into an archive file; HiP-HOPS can read both the full XML file and the packaged zip file. The elements in HiP-HOPS XML are discussed in the previous section.

The DOM parser is used to produce the file. HiP-HOPS elements are systematically represented as the XML elements and hierarchically appended/ structured.

\section{Case Study}

This section presents an example of a small standby system modelled using EAST-ADL. The simple architecture aims to illustrate the transformation process and the safety analysis capabilities obtained from the transformation. This system contains a pInputs component which provides inputs (this could be sensor reading for example) for the system, a primary component pPrimary which processes the inputs and produces a certain output or performs a function, and a standby component $\mathrm{pStandby}$ which performs identical function as pPrimary. These components both contain a subcomponent called ps111. The output from pPrimary and pStandby are supplied to another component called pCombiner. In a normal working condition, pCombiner will process and produce output based on information received from pPrimary. When a failure is detected on pPrimary (for example, absence of its input to pCombiner), pCombiner switches to use information from pStandby instead. 
Figure 4. Standby System Model

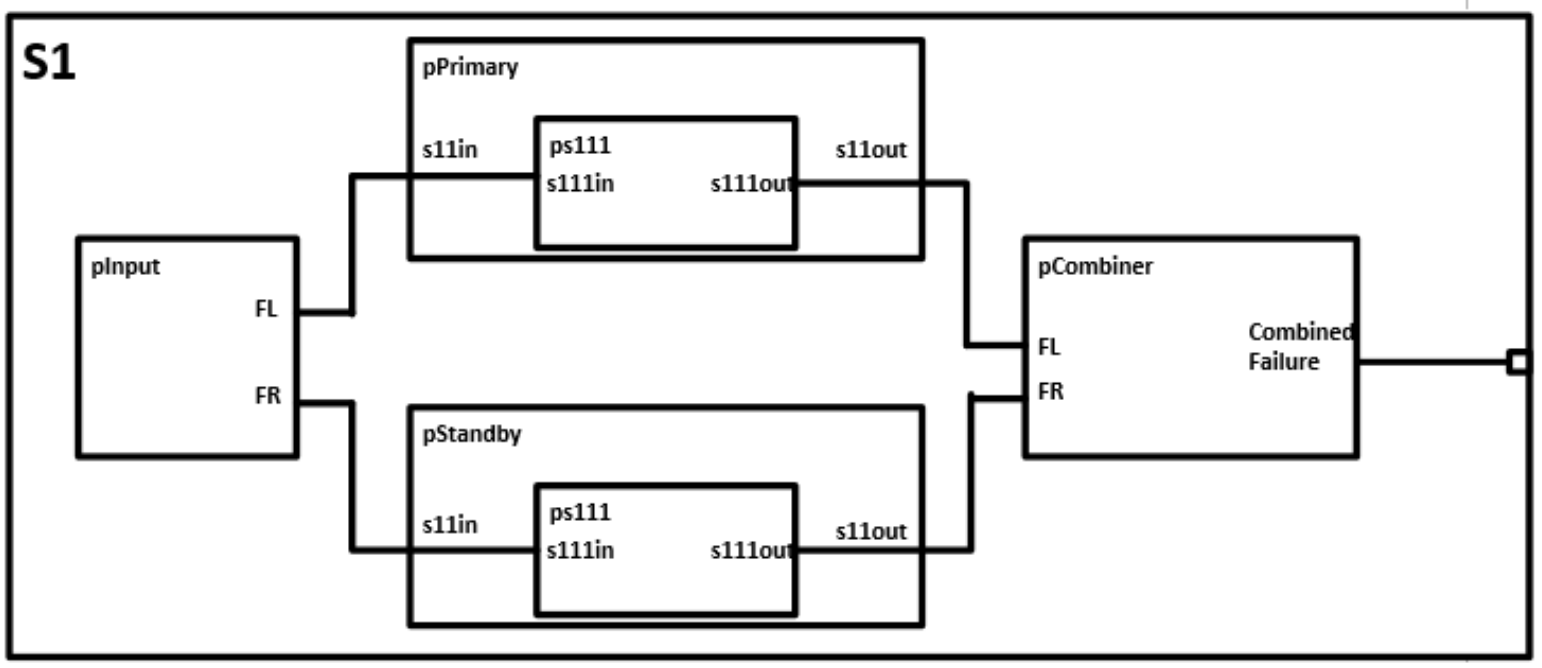

In EAST-ADL, this is modelled through top-level error model S1, which contains prototypes pInputs, pPrimary, pStandby, and pCombiner. These prototypes are instantiated from other error models. pInputs is instantiated from Inputs, pPrimary and pStandy are instantiated from s11, and pCombiner is instantiated from Combiner. As s11 contains subcomponent s111, prototypes pPrimary and pStandby contain prototype ps111. The following figure illustrates the EAST-ADL system hierarchy:

Figure 5. EAST-ADL Model of the System

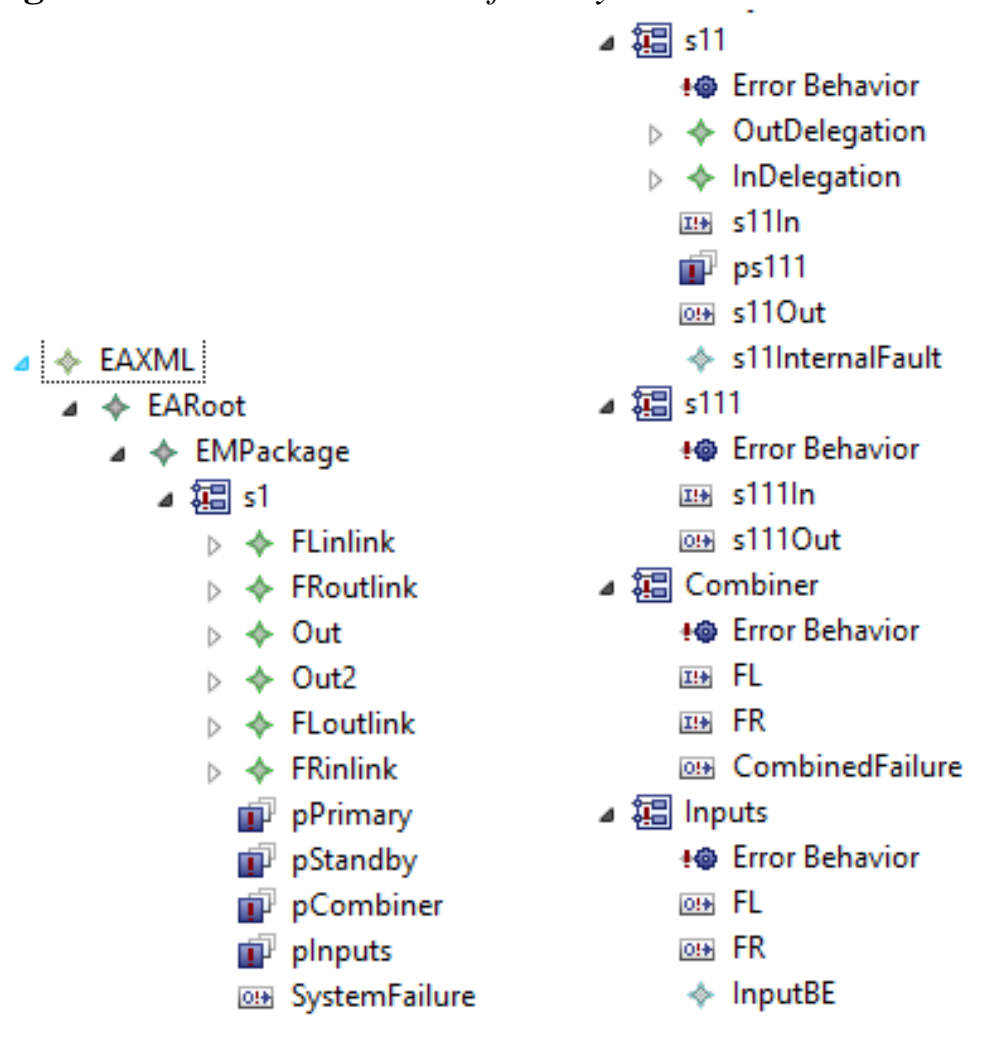


Information on the corresponding error behaviour has been included in the model. The following table summarizes the internal faults and failure logics for each of the error models. The failure logic defines how failures in the output can happen in relation to the internal fault and failure in the inputs. To maintain simplicity, each error model is assigned to have one internal fault. Output failure in Inputs is caused directly by its internal fault. The output failure in s11 and s111 can be caused by either internal fault or propagated input. The output failure in Combiner can be caused by failure in the input it receives from FL together with either a monitoring fault (causing the failure to be undetected and standby not activated) or failure of its FR input. An error model prototype inherits the error behaviour of the corresponding error model it was instantiated from. SystemFailure is directly linked to Failure-CombinedFailure in pCombiner.

Table 2. List of Component Internal Faults and Failure Logics

\begin{tabular}{|l|l|l|}
\hline Component & Internal Fault & Failure Logic \\
\hline s11 & s11InternalFault & $\begin{array}{l}\text { Failure-s11Out = Failure- } \\
\text { s11In OR s11InternalFault }\end{array}$ \\
\hline s111 & s111InternalFault & $\begin{array}{l}\text { Failure-s111Out = Failure- } \\
\text { s111In OR } \\
\text { s111InternalFault }\end{array}$ \\
\hline \multirow{3}{*}{ Combiner } & MonitoringFault & $\begin{array}{l}\text { Failure-CombinedFailure = } \\
\text { Failure-FL AN } \\
\text { (MonitoringFault OR } \\
\text { Failure-FR) }\end{array}$ \\
\hline Inputs & Failure-FL= InputBE; \\
& Failure-FR = InputBE; \\
\hline
\end{tabular}

This model has been automatically translated into a HiP-HOPS model through the translator tool. The hierarchy between prototypes are flattened and information on the error behaviour is included in the HiP-HOPS model in the appropriate format.

Once the HiP-HOPS model is constructed and annotated with failure information, FTA and FMEA can be performed. Figire 6 displays the resulting fault tree. The analysis results of the FTA is a list of minimal cut sets (as shown in figure 7) which identify the combination of component failures which cause the top-level failure. This list of minimal cut sets allows safety analysts to quickly identify single-point failures (in this case, the internal fault inputBE in Inputs is a single point of failure). Single point failure(s) are usually unacceptable in safety-critical systems. Critical or undesired combinations also highlight weak points in the system design. Their early identification subsequently allows these weak points to be targeted and addressed. For example, a design modification can be made to introduce fault-tolerant mechanisms, or a design decision can be made to use high-reliability implementations on critical components.

Although the structure of this example is small, and the minimal cut sets are relatively straight forward, the nature of the integration allows the 
automated benefits to be applied in larger, more complex systems, where manual analyses are often not feasible.

Figure 6. Fault Tree for Standby System

M FaultTrees

[F] Failure-s1.pCombiner.CombinedFailure

$\exists \cdot$ Failure-s1.pCombiner.CombinedFailure ()

$\exists \cdot$ Failure-s1.pCombiner.CombinedFailure (64)

$\square$ AND [Failure-s1.pCombiner.CombinedFailure] (35)

A OR [Failure-s1.pPrimary.s110ut] (114)

$\bigcirc$ s1.pPrimary.s11InternalFault (1)

s1.pInputs.InputBE (46)

s1.pPrimary.ps111.s111InternalFault (8)

$\square$ OR [Failure-s1.pCombiner.CombinedFailure] (37)

s1.pCombiner.MonitoringFault (33)

s1.pStandby.s11InternalFault (17)

s1.pInputs.InputBE (46)

s1.pStandby.ps111.s111InternalFault (24)

Figure 7. Minimal Cut Sets from FTA

$\bigcirc$ s1.pInputs.InputBE (46)

s1.pPrimary.s11InternalFault (1)

s1.pStandby.s11InternalFault (17)

s1.pPrimary.s11InternalFault (1)

s1.pStandby.ps111.s111InternalFault (24)

s1.pPrimary.s11InternalFault (1)

s1.pCombiner.MonitoringFault (33)

s1.pPrimary.ps111.s111InternalFault (8)

s1.pStandby.s11InternalFault (17)

s1.pPrimary.ps111.s111InternalFault (8)

s1.pStandby.ps111.s111InternalFault (24)

s1.pPrimary.ps111.s111InternalFault (8)

s1.pCombiner.MonitoringFault (33)

\section{Conclusions}

With the increasing importance of model-based system design in modern engineering, there is a growing demand for an integrated safety analysis within 
the development tool chain. This paper investigated the translation between the domain-specific modelling language EAST-ADL and the HiP-HOPS advanced dependability analysis tool.

The transformation between the two models was performed on tool level via the corresponding EAXML and HiP-HOPS XML interfaces. A translator tool has been developed, the transformation algorithm between the models was discussed, and a small example to demonstrate the transformation has been presented in this paper.

The work presented here enables various capabilities of HiP-HOPS, e.g. dependability analyses (i.e. safety, reliability, and availability) to be performed on automotive system models constructed in EAST-ADL. This benefits the system design and development by enabling the iterative and incremental safety analysis process throughout the lifecycle.

Future work in this area include improving the translator tool with automated model transformation techniques, for example, by using Eclipsebased ATLAS Transformation Language (ATL) ((Jouault, et al., 2008), or Epsilon Transformation Language (Kolovos, et al., 2008). Work is also in progress to enable seamless integration to the larger EAST-ADL Tool Platform, EATOP (Reiser \& Voget, 2012).

\section{References}

Adachi, M. et al., 2011. An Approach to Optimization of Fault Tolerant Architectures using HiP-HOPS. In: Software Practice and Experience. s.l.:John wiley \& Sons, Ltd, pp. 1303-1327.

Arnold, A., Gerald, P., Griffault, A. \& Rauzy, A., 2000. The Altarica Formalism for Describing Concurrent Systems. Fundamenta Informaticae, Volume 34, pp. 109124.

AUTOSAR, 2013. [Online] Available at: http://www.autosar.org/ [Accessed 20 July 2014].

Biehl, M., DeJiu, C. \& Torngren, M., 2010. Integrating Safety Analysis into the Model-based Development Toolchain Embedded Systems. LCTES, pp. 125-131.

Blom, H., 2013. EAST-ADL XML Schema, s.l.: s.n.

Blom, H. et al., 2012. EAST-ADL- An Architecture Description Language for Automotive Software- Intensive Systems, s.l.: s.n.

Bozzano, M. \& Villafiorita, A., 2006. The FSAP/NuSMV Safety Analysis Platform. Internal Journal on Software Tools for Technology Transfer, Volume 9, pp. 5-24.

Chen, D. et al., 2013. System Modelling with EAST-ADL for Fault Tree Analysis through HiP-HOPS. York, s.n.

EAST-ADL, 2011. EAST-ADL Domain Model Specification, Vesion M.2.1.9. [Online] Available at: www.maenad.eu [Accessed 2007 2014].

EAST-ADL, 2014. EAST-ADL Domain Model Specification, Vesion M.2.1.12. [Online] Available at: www.east-adl.info/Specification.html [Accessed 3007 2014].

Joshi, A., Heimdahl, M. P., Miller, S. P. \& Whalen, M. W., 2006. Model-Based Safety Analysis, Hampton: NASA Langley Research Centre.

Jouault, F., Bezivin, J. \& Kurtev, I., 2008. ATL: a Model Transformation Tool . In: Science of Computer Programming. s.1.:s.n., pp. 31-39. 
Kolovos, D., Paige, R. \& Polack, F., 2008. The Epsilon Transformation Language. In: Theory and Practice of Model Transformations. s.l.:s.n., pp. 46-60.

Mian, Z. et al., 2014. Model Transformation for Multi-Objective Architecture Optimization of Dependable Systems. In: Dependability Problems of Complex Information Systems. s.1.:Springer, pp. 91-110.

Papadopoulos, Y., McDermid, J., Sasse, R. \& Heiner, R., 2001. Analysis and Synthesis of the Behaviour of Complex Programmable Electronic Systems in Condition of Failure. Reliability Engineering and System Safety, Volume 71, pp. 229-247.

Reiser, M. \& Voget, S., 2012. EATOP-EAST-ADL Tool Platform, s.l.: s.n.

Sharvia, S. \& Papadopoulos, Y., 2011. Integrated Application of Compositional and Behavioural Safety Analysis. Dependability and Complex System, Volume 97, pp. 179-192.

Walker, M., Parker, D. \& Papadopoulos, Y., 2013. HiP-HOPS XML Input Format, s.l.: University of Hull. 